\title{
The hadronic interaction model Sibyll - past, present and future
}

\author{
Ralph Engel ${ }^{1, \text { a }}$, Felix Riehn ${ }^{1,2,4, \mathrm{~b}}$, Anatoli Fedynitch ${ }^{3}$, Thomas K. Gaisser ${ }^{4}$, and Todor Stanev ${ }^{4}$ \\ ${ }^{1}$ Institute for Nuclear Physics (IKP), Karlsruhe Institute of Technology (KIT), P.O. Box 3640, 76021 Karlsruhe, Germany \\ 2 LIP, Av. Elias Garcia 14-1, 1000-149 Lisboa, Portugal \\ 3 DESY, Platanenallee 6, 15738 Zeuthen, Germany \\ ${ }^{4}$ Bartol Research Institute \& Department of Physics and Astronomy, University of Delaware, Newark, DE 19716, USA
}

\begin{abstract}
Sibyll is one of the first microscopic interaction models that was specifically developed for interpreting cosmic ray data. It combines non-perturbative concepts of simulating hadronic particle production with predictions derived from perturbative QCD calculations, focusing on forward particle production of relevance in studying cosmic ray interactions. In this contribution we briefly recall the history of Sibyll and then, in this context, describe improvements made in the different versions of the Sibyll model. The discussion focuses on the basic concepts and ideas of these improvements rather than going into detail or giving a comprehensive description of the models. We also discuss shortcomings, conceptual problems, and uncertainties in modeling hadronic interactions and make some suggestions how to address these open questions in the future.
\end{abstract}

\section{Introduction}

In the early days of cosmic ray physics the measurements of inclusive fluxes and air showers were compared to analytic and semi-analytic calculations to derive information on the energy of the primary particles, their flux, composition, and also to learn about general features of hadronic multiparticle production. A system of coupled cascade equations had to be solved for different initial conditions (see, for example, [1-4]). A number of approximations and simplifications were needed to keep the problem of calculating secondary particle fluxes and particle cascades treatable at the time. With the availability of increasingly powerful computers, solving cascade equations numerically became more and more common [5-7]. But even then it was not possible to calculate the secondary fluxes in full detail, and showerby-shower fluctuations could not be predicted. Ultimately, the full complexity of the problem can only be treated with Monte Carlo methods, which are suited to deal with the large number of degrees of freedom involved in these calculations.

Today a variety of dedicated program packages is publicly available for calculating the results of cosmic ray interactions in the atmosphere. These include programs in which Monte Carlo generated tables of hadronic interactions are used to numerically solve cascade equations (for example, MCEq [8]), programs in which the Monte Carlo method is combined with numerical solutions of cascade equations (for example, SENECA [9] and CONEX [10]), and fully Monte Carlo based programs (for example, AIRES [11,12], CORSIKA [13], and COSMOS [14]).

\footnotetext{
a e-mail: ralph. engel@kit.edu

b e-mail: friehn@lip.pt
}

Very important elements of these code packages are hadronic interaction models, often referred to as event generators. Similar to the evolution found in cascade calculations, the first interaction models were just analytic parametrizations of the secondary particle yields and phase space distributions. One of the notable very early Monte Carlo models of hadronic interactions is the Hillas splitting algorithm (see [15] and also appendix of [4]), which was developed for one of the first Monte Carlo packages for air showers, MOCCA $[15,16]$. This splitting algorithm is still used in a slightly modified form in AIRES. With time the complexity of hadronic interaction models increased quickly and we now have very sophisticated and powerful Monte Carlo event generators available. These event generators are based on microscopic models and certain aspects of QCD, the theory of strong interactions, and represent the state-of-the art of our understanding of high-energy hadronic multiparticle production. Sibyll [17-21] is one of these event generators whose development started already in the late 1980ies.

After recalling the motivation for developing Sibyll we will discuss the major steps of arriving at the currently available version 2.3 of this interaction model (Sect. 2). Recent improvements triggered by the progress in understanding hadronic interactions in general, and the new LHC and fixed-target measurements in particular, will be summarized in Sect. 3. After presenting examples of comparisons to accelerator data, predictions for air showers and inclusive fluxes will be shown and discussed in the context of those obtained with other interaction models. Finally, a critical outlook is given in Sect. 4.

\section{The past - a short history of Sibyll}

In the following we will recall some fundamental hypothesis and experimental observations to introduce the 
scientific contest and the motivation for developing the Sibyll model.

\subsection{Regge theory}

In the early days of high energy physics it was common wisdom that the total and elastic interaction cross sections of hadrons decrease with the center-of-mass energy $\sqrt{s}$. This behavior was well understood in terms of Regge theory [22] by assuming maximally analytic scattering amplitudes and accounting for exchanges of many hadronic particles in a scattering process. Qualitatively, the predicted relation is $\sigma \sim s^{\alpha_{i}(0)-1}$, with $\alpha_{i}(0)$ being a constant, called the Regge intercept of the Regge trajectory $\alpha_{i}(t)$, which depends on the quantum numbers of the exchanged hadrons. These Regge intercepts are related to the spin-mass relation of resonances (Chew-Frautschi plot [23]) and the largest measured values of $\alpha_{i}(0)$ are in the range of $0.45-0.55$.

With the first data from the Serpukhov accelerator it became clear that this seemingly self-consistent picture, based entirely on hadronic degrees of freedom, had to be extended. The Serpukhov data of the late 1960ies indicated that hadronic cross sections start to be energy-independent above $\sim 50 \mathrm{GeV} / \mathrm{c}$ lab. momentum $(\sqrt{s} \sim 10 \mathrm{GeV})$. This motivated Pomeranchuk to postulate the existence of a new Regge trajectory with $\alpha(0) \approx 1$, now commonly referred to as the pomeron. In 1971, with the data of the CERN Intersecting Storage Ring (ISR), it became obvious that the total and elastic cross sections of hadrons slowly increase with energy. In the Reggeon model, this can only be explained by assuming a super-critical pomeron, i.e. $\alpha(0)>1$. Since then there have been many attempts to find the resonances belonging to the pomeron trajectory, which are possibly short-lived glue balls. Whether glue balls indeed exist is still an open question [24].

At this time also a number of phenomenological scaling hypotheses were commonly tested and found to be approximately satisfied. The most important ones are Feynman scaling [25] of particle production cross sections and Koba-Nielsen-Olesen (KNO) scaling [26] of particle multiplicities.

If Feynman scaling applies, the inclusive production cross section $a b \rightarrow c X$ is expected to approach, in the high-energy limit, a universal function

$$
E \frac{\mathrm{d} \sigma_{a b \rightarrow c}}{\mathrm{~d}^{3} p} \rightarrow f_{a b \rightarrow c}\left(x_{F}, p_{\perp}\right),
$$

with $x_{F}=p_{\|} / p_{\|, \max } \approx p_{\|} / \sqrt{s}$ and $p_{\perp}$ being the transverse momentum of $c$. An important consequence of Feynman scaling is the prediction of an energyindependent height of the rapidity distribution $\mathrm{d} N_{a b \rightarrow c} / \mathrm{d} y$ of particle $c$ for $x_{F} \rightarrow 0$

$$
E \frac{\mathrm{d} \sigma_{a b \rightarrow c}}{\mathrm{~d}^{3} p}=\frac{\mathrm{d} \sigma_{a b \rightarrow c}}{\mathrm{~d} y \mathrm{~d}^{2} p_{\perp}} \rightarrow f_{a b \rightarrow c}\left(x_{F} \rightarrow 0, p_{\perp}\right) .
$$

Another important phenomenological scaling hypothesis, which is similar to that of Feynman scaling, is the idea limiting fragmentation [27]. Here the assumption is that the distributions of leading particles, i.e. particles with momenta similar to those of the projectile or target, depend only on the projectile and target particles and approach a universal function at very high energy.
KNO scaling manifests itself by energy-independent moments, $\gamma_{q}$, of the multiplicity distribution

$$
\gamma_{q}=\frac{\left\langle(n-\bar{n})^{q}\right\rangle}{\bar{n}^{q}},
$$

where $\bar{n}$ denotes the average particle multiplicity and the average is taken over all events of the data sample.

All these phenomena could be reasonably well described, on a phenomenological level, by Regge-inspired models. The energy dependence of the total and elastic cross sections could be parametrized and, with the help of the triple-Regge graph [28] and corresponding unitarity cuts, it was even possible to understand inclusive particle distributions. Still, most of the predictions were of a qualitative nature and measurements were needed to make them more quantitative.

\subsection{Parton model and perturbative QCD}

After the introduction of quarks for the classification of hadrons in $1964[29,30]$ the concept of constituents of hadrons was extended to high-energy scattering phenomena within the parton model. Originally, the parton model was developed by Feynman [31-33]. to describe scaling laws found in inelastic lepton-nucleon scattering by introducing point-like constituents in hadrons, but soon many other applications were found. Finally, with the formulation of Quantum Chromodynamics (QCD) [34] in 1973 a selfconsistent theory of strong interactions was available.

QCD was enthusiastically adopted with the realization that asymptotic freedom of partons is one of its fundamental properties. It was realized that Feynman's partons were the quarks and gluons of QCD and soon first predictions of parton-parton scattering, calculated in perturbation theory $[35,36]$, and estimates for high- $p_{\perp}$ particle and jet production [37] were made and found to be in reasonable agreement with measurements. Also scaling laws for the fragmentation of partons to (leading) hadrons were derived [38] and evolution equations for the densities of partons in hadrons were written down [39].

In the early 1980ies rare processes involving large momentum transfer (hard processes), such as jet production, could be calculated reliably and many experiments at colliders were built to study these processes. At the same time, very little progress was made in understanding the bulk of hadronic particle production that does not proceed through processes of high momentum transfer (soft processes). Even though the partonic view of highenergy interactions was very successful for hard processes, Regge-type models were still needed to describe soft hadron production processes. In particular, it became clear that it is very difficult to combine the hadronic view of soft processes with the partonic view of hard processes in a consistent and quantitative way [40].

\subsection{Minijet Model}

In the second half of the 1980ies the data of the CERN SPS collider confirmed the growth of the total and elastic $p \bar{p}$ cross sections $[41,42]$ as already seen at the ISR. New observations were the strong violation of Feynman scaling at central pseudorapidities [43] and also the violation of KNO scaling [44]. For example, the pseudorapidity 


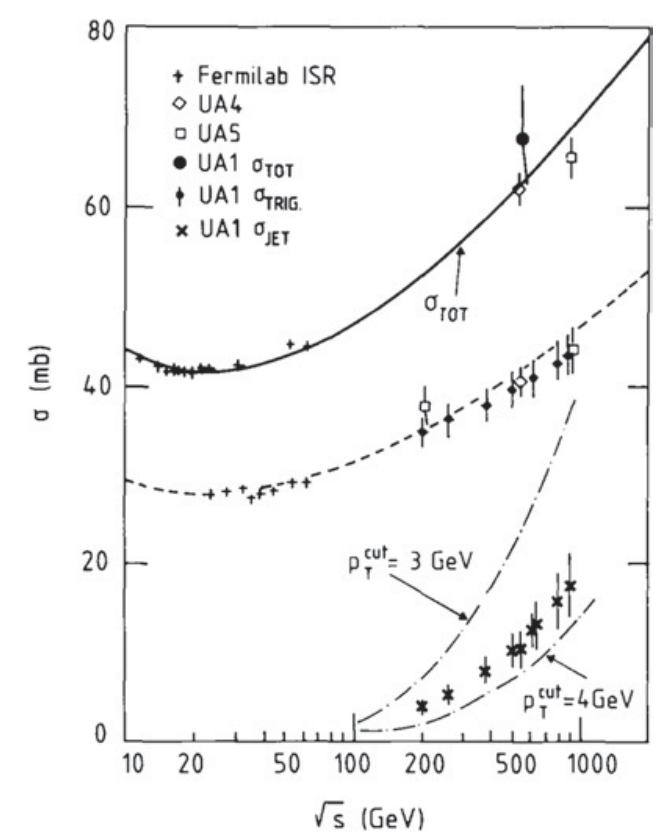

Figure 1. Inclusive cross section for minijet production measured by the UA1 Collaboration [46]. The data are compared to measurements and model curves for the elastic and total $\bar{p} p$ cross sections. Also shown are predictions based on perturbative QCD for parton-level jets for different transverse momentum thresholds (dash-dotted lines).

density of charged particles $\mathrm{d} N_{\mathrm{ch}} / \mathrm{d} \eta$ changes from 2.2 at $\sqrt{s}=200 \mathrm{GeV}$ to about 3 at $\sqrt{s}=900 \mathrm{GeV}$ for $\eta \approx 0$. In contrast, the leading particle distributions, as far as accessible at the SPS experiments, were found to scale as expected for limiting fragmentation $[43,45]$.

Another surprising result was the rapid increase with energy of the inclusive cross section for jet production [46]. This is shown in Fig. 1. This observation led to a controversial discussion about down to what scales of momentum transfer can perturbative calculations still be expected to be reliable. Calculations of cross sections for so-called minijet production (i.e. jets with a transverse momentum of $3-5 \mathrm{GeV}$ ) were met with scepticism and the debate is still ongoing.

Extrapolating the measured jet rates or relying on calculated inclusive cross sections led to the conclusion that hard parton-parton scattering should be an abundant process in hadronic scattering at high energy [47]. Therefore it was very natural to assume that, if the untilthen theoretically unexplained rise of the total cross section does originate from minijet production, a new process is setting in just in this energy range. After all, parton-parton scattering is a fundamental and undisputed prediction of QCD.

One of the key problems with this assumption was, however, the fact that the cross section for jet production increases with energy much faster than the total cross section, see Fig. 1. This problem is addressed in the Minijet Model [48-52], which was developed in the late 1980ies. It was the first model offering a consistent microscopic picture of the relation between the inclusive jet production rate and the growth of the elastic and total cross sections.

In the minijet model, the apparent contradiction between the rapid growth of the inclusive jet cross section and the only moderate growth of the total cross section

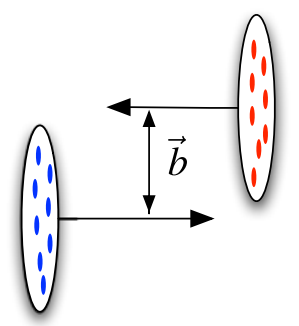

Figure 2. Geometric picture of two hadrons colliding at high energy. The hadrons are Lorentz-contracted along the beam axis. The distribution of partons in a hadron is described by their density in transverse space. The number of parton pairs expected to interact depends on the parton densities in transverse space and the impact parameter $|\mathbf{b}|$ of the collision.

is resolved by allowing for multiple, independent parton pairs interacting in a single hadronic collision. Keeping in mind that jet cross sections $\sigma_{\text {jet }}$ calculated within perturbative QCD are inclusive cross sections one has the relation

$$
\sigma_{\text {jet }}=\left\langle n_{\text {int }}\right\rangle \sigma_{\text {ine }},
$$

with $\left\langle n_{\text {int }}\right\rangle$ being the average number of interacting parton pairs and $\sigma_{\text {ine }}$ denoting the inelastic cross section. If, for simplicity of the argument, the energy dependence is written as $\sigma_{\text {ine }} \sim s^{0.08}$ and $\sigma_{\text {jet }} \sim s^{0.4}$ a rapid growth of the mean number of interactions $\left\langle n_{\text {int }}\right\rangle \sim s^{0.32}$ is predicted. Already a very simple and intuitive geometric model of hadrons is sufficient to calculate the probability distribution of the number, $n_{\mathrm{int}}$, of interacting parton pairs and, hence, the relation between the inelastic and jet cross sections. This is illustrated in Fig. 2. Neglecting possible correlations between the partons in the colliding hadrons (the partonic state is frozen-in for the time of the hadronic collision) one obtains the well-known eikonal model for multiple interactions (for a pedagogical presentation, see [4]). The eikonal formulas can also be derived within field theory. This more rigorous derivation also predicts the correct complex phases for the resulting scattering amplitudes, see $[53,54]$ and references therein.

The minijet model, combined with the eikonal approach for estimating the amount of multiple partonic interactions, is a model that has only a very small number of parameters. These are the transverse momentum threshold that is needed for regarding partonic interactions as hard processes to which asymptotic freedom applies (and also for calculating the inclusive minjet cross section), the parton density functions $f\left(x, Q^{2}\right)$, and the parton distributions in transverse space. To make the model complete soft interactions have to be added, which cannot be calculated in perturbation theory.

There are a number of very remarkable successes of the minijet model $[48,55]$. First of all, the rise of the observed cross sections can be understood and predictions for higher energies can be calculated, see [50,56-60] for examples of early works. Secondly, the multiplicity distribution of secondary particles, including the increasing violation of KNO scaling is correctly predicted $[48,61,62]$. Thirdly, the model predicts a rise of the central pseudorapidity plateau and, hence, violation of Feynman scaling at $\left|x_{F}\right| \approx$ 0 . Additional features of the model are strong forwardbackward multiplicity correlations in rapidity space [63] and the expectation of only very minor scaling violations 
of the distributions of leading secondary particles, which are related to effects of energy-momentum conservation.

These successes of the minijet model are, to a large extent, related to the concept of multiple interactions [64]. Other multiple interaction models, such as the Dual Parton Model [65] and the Quark-Gluon Strings Model [66], which were originally developed for soft interactions only, also describe many of the observed features of multiparticle production up to $\sqrt{s} \sim 200 \mathrm{GeV}$. In comparison to these models, the minijet model is an attempt to relate as many features of multiparticle production as possible to hard parton-parton interactions and to minimize additional assumptions needed for soft processes. With hard interactions becoming increasingly important at high energy, this approach promises a framework for extrapolations to very high energies without many parameters.

\subsection{Early versions of Sibyll up to Sibyll 1.7}

Already three years after the development of the minijet model, a first implementation of multiple hard interactions in the hadronic event generator PYTHIA became available in 1987 [67]. In PYTHIA, only the cross section of inelastic events with (possibly multiple) hard partonparton interactions is calculated following the minijet model. This cross section is considered as a part of the larger total inelastic cross section, which is taken from an external parametrization. No attempt is made to fully implement the minijet model, i.e. to also derive the total and elastic cross sections from the implemented partonic cross sections.

Sibyll was the first Monte Carlo event generator with a full implementation of the minijet model. Elastic and total cross sections as well as partial cross sections for a given number of multiple interactions are calculated in a self-consistent way. Key assumptions of the model were a constant soft cross section, which had to be added in the eikonal to the inclusive cross section for hard interactions, and the assumption of an energy-independent transverse momentum threshold $p_{\perp}^{\text {cut }}$ for calculating the minijet cross section. Many aspects of Sibyll are very strongly inspired by the Lund approach for modeling hadronic interactions. The string fragmentation used in Sibyll, for example, is an implementation of the Lund model $[68,69]$ of these days.

Early versions of the code date back to 1987 and were used in various research projects, for example, see [70]. In a collaboration with J. Engel, a library for nuclear targets and projectiles [17] was developed in the early 1990ies, adding a sophisticated abrasion-ablation model for nuclear fragmentation to Sibyll. Proton/pion/kaon interactions with the nuclei of air are simulated based on the Glauber model for nuclear interactions [71]. Keeping in mind the application for air shower simulations, the interaction of primary nuclei is implemented using the semi-superposition model. This means that the depth distribution of interaction points, the number and mass distribution of the nuclear fragments, and number of interacting nuclei is correctly calculated using the Glauber approach combined with a nuclear fragmentation model. But at each given interaction point $N$ interacting nucleons of the projectile are replaced by $N$ protons and neutrons whose interaction with air nuclei is simulated separately [17]. Finally, version 1.5 of Sibyll was made publicly available and described in detail in Ref. [18]. Minor additions such as including neutral pions as possible projectiles led to an increase of the version number to 1.7. In 1993 Sibyll was included as one of the first microscopic interaction models in the shower simulation code MOCCA [15,16], and later CORSIKA [13], and AIRES [11,12]. A multitude of shower simulations were done with Sibyll, including all the simulations for designing the Pierre Auger Observatory [72,73].

\subsection{Sibyll 2.1}

Although Sibyll 1.7 was a very successful model in the 1990ies, it was far from being perfect.

Already the comparison to data of fixed-target and collider experiments made in the initial publication of Sibyll showed some shortcomings of the model [18]. For example, the predicted distribution of the secondary particle multiplicity was not as wide as found in data. Other problems were related to the overall growth of the particle multiplicity with energy and the distribution of secondary particles around $x_{F} \sim 0.8-0.9$, the transition region between non-diffractively and diffractively produced secondaries.

Independent of the discrepancies found in comparisons with accelerator data, the first data on deep-inelastic scattering of the HERA collider indicated a behavior of parton densities at low $x$ similar to $f\left(x, Q^{2}\right) \sim x^{-1.3}$, which is very different from what had been commonly used before. The minijet cross section in Sibyll 1.x had been calculated with the Eichten-Hinchliffe-LaneQuigg [74] (proton) and Duke-Owens [75] (pion) parton densities, which satisfy $f\left(x, Q^{2}\right) \sim x^{-1}$.

Last but not least, there were also indications from the KASCADE air shower array [76] that the showers simulated with Sibyll either had too many electrons or too few muons at ground level for obtaining a consistent description of shower data through the knee energy region.

These observations triggered the development of an improved version of Sibyll that started in 1997. The model for diffraction dissociation was replaced by a more consistent treatment of diffraction using a two-channel eikonal model based on the Good-Walker approach [77]. Multiple soft interactions were implemented in the generation of the string configurations and hadronic final states to consistently interpret the eikonal amplitude according to the Abramovski-Gribov-Kancheli cutting rules [78]. The parametrizations of parton densities were updated to those by Glück, Reya, and Vogt $[79,80]$, which were in good agreement with HERA data at this time.

The modified parton densities led to a much steeper increase of the predicted minijet cross section and it became clear that, even by adjusting model parameters very generously, the slow rise of the total cross section could not be described without modifying the original ideas of the minijet model. Either the total cross section would grow too fast or the secondary particle multiplicity would exceed by far that found at the Tevatron. Qualitatively, the correlation of these quantities follows directly from Eq. (4). An eikonal model satisfies unitarity, i.e. the inclusive cross section for minijet production always stays the same.

Unitarity is such a fundamental property of scattering amplitudes that the tension between the energy dependence 
of the jet cross section, total cross section, and the particle multiplicity has to be interpreted as a failure to calculate inclusive minijet cross section (the other quantities are measured, and (4) holds in any model that satisfies unitarity). There are many reasons why a calculation at transverse momenta as low as $p_{\perp} \sim 2-3 \mathrm{GeV} / \mathrm{c}$ could fail. For example, collinear factorization as used in most perturbative calculations is expected to break down and, instead of leading $\log \left(Q^{2} / \Lambda_{\mathrm{QCD}}^{2}\right)$ terms, one should re-sum $\log (1 / x)$ terms, or the extrapolation of parton densities to low $x$ is failing for such low parton virtualities. Also calculations at leading-log accuracy are know to be subject to large uncertainties stemming from the arbitrary choice of the factorization and renormalization scales.

Such problems in calculating the minijet cross section had already been anticipated many years earlier, see [47] for a review. The large density of partons at low $x$ leads to collective effects, i.e. partons cannot be considered to be independent objects anymore. Whether these collective effects lead to a saturation or just a reduction of the parton densities is the subject of an ongoing debate.

In the high-energy limit, i.e. $\ln (1 / x), \ln \left(Q^{2} / \Lambda_{\mathrm{QCD}}^{2}\right) \rightarrow$ $\infty$ (double-leading-log approximation), the gluon density $g\left(x, Q^{2}\right)$ is given by

$$
x g\left(x, Q^{2}\right) \sim \exp \left[\frac{48}{11-\frac{2}{3} n_{f}} \ln \frac{\ln \frac{Q^{2}}{\Lambda_{\mathrm{QCD}}^{2}}}{\ln \frac{Q_{0}^{2}}{\Lambda_{\mathrm{QCD}}^{2}}} \ln \frac{1}{x}\right]^{\frac{1}{2}} \sim \frac{1}{x^{0.4}},
$$

with $n_{f}$ being the number of active quark flavors. Then, assuming a transverse size of a gluon being about the size of its cross section $\sigma \sim \alpha_{s}\left(p_{T}^{2}\right) / p_{\perp}^{2}$, one has as condition for all gluons fitting next to each other in a proton of radius $R_{p}[81]$

$$
\frac{\alpha_{s}\left(p_{T}^{2}\right)}{p_{T}^{2}} \cdot x g\left(x, p_{T}^{2}\right) \leq \pi R_{p}^{2},
$$

where $\alpha_{s}$ is the strong coupling constant. If the gluons are not distributed democratically in transverse space by forming, for example, hot spots around the valence quarks, the non-linear effects suppressing the number of independent partons will set in much earlier than indicated by Eq. (6).

In Sibyll, Eq. (6) is used as guidance for introducing an energy-dependent transverse momentum threshold applied in calculating the minijet cross section

$$
p_{\perp}^{\min }(s)=p_{\perp}^{0}+\Lambda \exp \left[c \sqrt{\ln \left(s / \mathrm{GeV}^{2}\right)}\right]
$$

with $p_{\perp}^{0}$ and $\Lambda$ being model parameters. All interactions producing only partons with $p_{\perp}<p_{\perp}^{\min }(s)$ are considered as soft interactions. The previously constant cross section for soft interactions had to be made energy-dependent to account for the fraction of partonic interactions that has been shifted in the classification from hard to soft. Inspired by Regge phenomenology for soft processes, this energy dependence is assumed to be a power law $\sigma_{\text {soft }} \sim s^{\Delta}$ with $\Delta$ being a parameter that depends on $p_{\perp}^{0}$ and $\Lambda$. $\Delta$ is determined by fitting total and elastic cross sections (typical values are $\Delta \approx 0.1$ ).

The fit to the the $p p$ and $p \bar{p}$ cross sections is shown in Fig. 3. Thanks to the improved model for diffraction

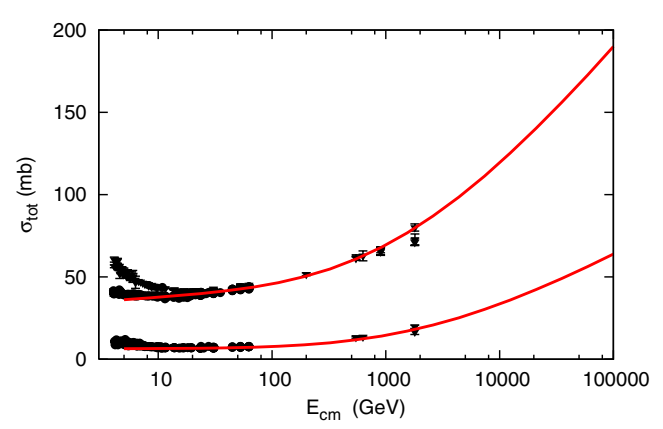

Figure 3. Elastic and total cross sections of $p p$ and $p \bar{p}$ interactions. Shown are data together with the cross section fit of Sibyll 2.1 [19] as a function of the center-of-mass energy $E_{\mathrm{cm}}=\sqrt{s}$.

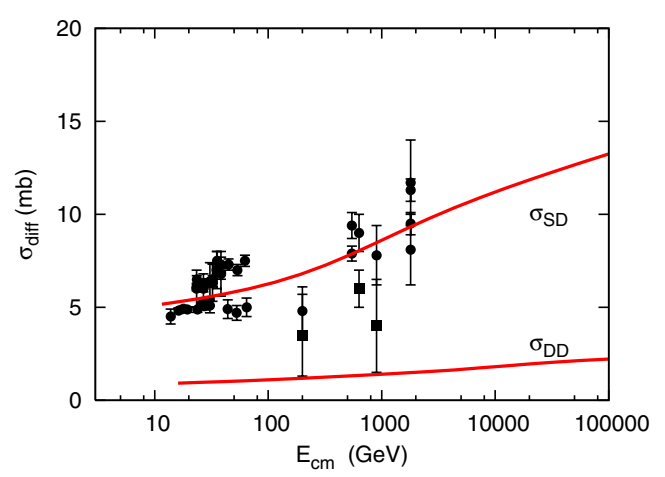

Figure 4. Single- and double-diffractive cross sections of $p p$ and $p \bar{p}$ interactions. Shown are measurements for diffractively produced masses of $M_{D}^{2}<0.05 s$ together with the predictions of Sibyll 2.1 [19] as a function of the center-of-mass energy $E_{\mathrm{cm}}=\sqrt{s}$.

dissociation, a good description of single- and doublediffractive cross sections is also obtained, see Fig. 4.

This construction of matching soft and hard interactions illustrates a generic problem of hadronic interaction models. Strictly speaking, partons are only well defined objects for hard interactions. Nevertheless, there has to be a smooth transition to soft interactions, for which the parton language does not apply. The currently applied makeshift solution is to generate string configurations for soft interactions that would correspond to hard interactions with very low $p_{\perp}$, i.e. two strings per interaction. Another problem is the significantly different energy dependence of the soft and hard processes. A smooth transition between the two categories of processes can only be maintained if the transverse momentum threshold for hard processes is increasing with energy.

Finally it should be mentioned that the inclusion of multiple soft interactions in Sibyll is nothing other than a particular implementation of Gribov's Reggeon calculus [82]. In this sense the model implemented in the new version of Sibyll goes beyond the classic minijet model of the late 1980ies and becomes in some aspects similar to the Dual Parton Model realizations in PHOJET $[83,84]$ and DPMJET $[85,86]$.

In 1999 first predictions of the new version of Sibyll were shown [90] and the code was released to the public in 2000 as Sibyll 2.1. Because of the conceptually non-satisfying treatment of the matching of soft and hard interactions this model was never considered as 

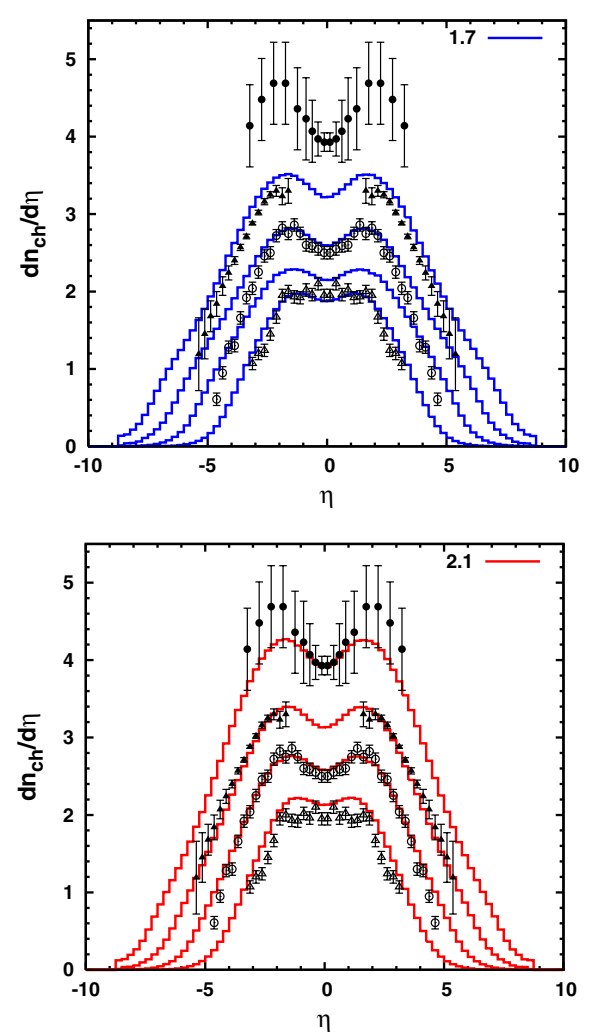

Figure 5. Pseudorapidity distribution of charged particles in $p \bar{p}$ collisions. ISR and SPS data are compared to predictions of Sibyll 1.7 and Sibyll 2.1 [19]. Data points from top are $\sqrt{s}=$ $1800 \mathrm{GeV}$ [87], $630 \mathrm{GeV}$ [88], $200 \mathrm{GeV}$ [43], and $53 \mathrm{GeV}$ [89].

a long-term version of Sibyll. The successes of the model in describing many collider measurements and also providing reasonable interpretations of air shower data showed, however, that the model was well-suited for the purposes it had been developed for. Motivated by the continuous use of the model in many calculations, a comprehensive description of the model was finally published in 2009 [19].

One of the very striking improvements in describing data when switching from Sibyll 1.7 to Sibyll 2.1 is shown in Fig. 5. While this figure shows data to which the model was tuned, predictions for higher energies are compared with first LHC measurements in Ref. [91]. The Sibyll extrapolations were very close to these measurements.

\section{The present - Sibyll version 2.3}

The very encouraging agreement of Sibyll predictions with the first minimum bias data of LHC experiments give strong support to the underlying ideas of this model, even though it is much less sophisticated than other hadronic interaction models such as EPOS [99-101], QGSJET [102-105], and DPMJET [85,86]. On the other hand, the LHC data led to the discovery of new shortcomings and helped reducing the uncertainties of the model extrapolation to high energy.

Motivated by the LHC data and also new fixedtarget measurements a number of improvements have been implemented in Sibyll. A new version of the code, Sibyll 2.3, was released in 2016. In the following an overview of some of the model changes are given. A complete

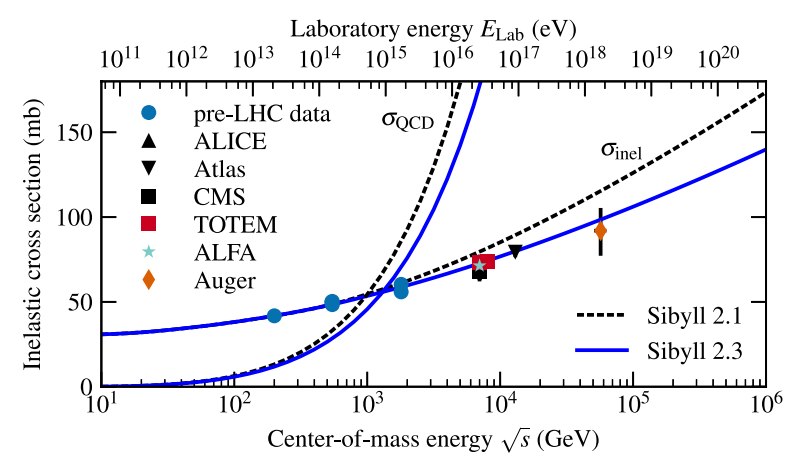

Figure 6. Inelastic cross section for $p \bar{p}$ interactions. Data are compared to calculations made with Sibyll versions 2.1 and 2.3. The new LHC measurements [92-97] are shown individually. At high energy the cross section derived from $p$-air interactions by the Auger Collaboration is also given [98]. In addition the inclusive cross section for minijet production of the two model versions is shown as dashed lines.

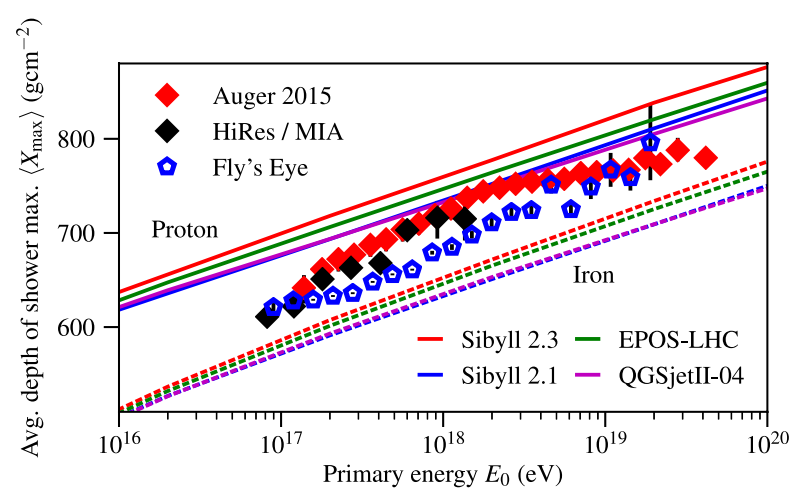

Figure 7. Mean depth of shower maximum. Data [110-112] are compared with different model predictions.

description of the model will be given in a forthcoming publication [106].

The discrepancy between the different measurements of the total $p \bar{p}$ cross section [107-109] at Tevatron led to a considerable uncertainty in the cross section extrapolation. The fit of Sibyll 2.1 made in 2000 was favoring the higher value of the CDF Collaboration, see Fig. 3. LHC data have greatly reduced this uncertainty and the Sibyll predictions were found to be too high. By changing the shape of the distribution of the partons in transverse space, see Fig. 2, the Sibyll cross section has been adjusted to fit the LHC data. A comparison of the previous and current cross section predictions of the Sibyll model is shown in Fig. 6. Although a reasonable description of the LHC data on elastic and total cross sections could be obtained, a deviation was found for the slope of the differential elastic cross section $\mathrm{d} \sigma / \mathrm{d} t$ at $|t| \rightarrow 0$. For a better description of elastic scattering a full treatment of the complex phase of the scattering amplitude is needed, which is beyond the scope of the Sibyll model.

Another important improvement of the model was the implementation of the Good-Walker approach [77] to inelastic diffractive scattering in form of a two-channel model also for interactions with nuclei. This lead to an increase of the cross section for projectile diffraction dissociation on nuclei. Together with the reduced inelastic cross section this results in a shift of the predicted mean depth of shower maximum deeper into the atmosphere, see Fig. 7. 


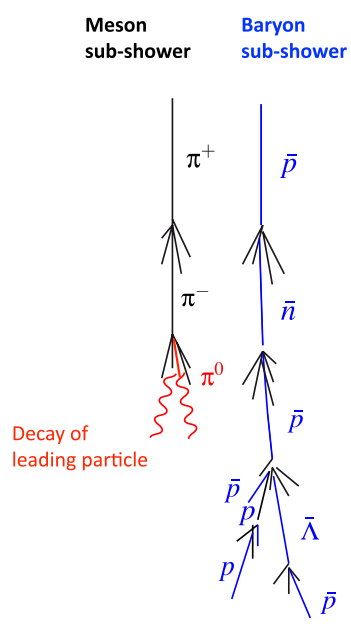

Figure 8. Illustration of processes of direct relevance to muon production in air showers. Right: Baryon number conservation makes baryons a very efficient source of secondary hadrons. Left: There is about a $30 \%$ chance probability that the leading particle of a pion-air interaction is a neutral pion. Hence subshowers initiated by charged pions have a high probability to be terminated by production of a leading $\pi^{0}$.

Further improvements of Sibyll 2.3 with respect to Sibyll 2.1 are the sampling of the transverse momentum of partons at the string ends from an exponential distribution in transverse mass $m_{\perp}=\sqrt{p_{\perp}^{2}+m^{2}}$ instead of $p_{\perp}$ and a better sampling of the momentum fractions of the partons entering hard scattering processes. These changes allow a better description of a multitude of LHC data sets, but are not expected to be of great importance for air shower predictions.

Air shower calculations with Sibyll always predicted a smaller number of muons at ground than one would obtain with EPOS or QGSJET, see [113]. A number of air shower measurements indicate, however, that even simulations with EPOS or QGSJET do predict fewer muons than observed in the data [114-117]. Therefore special emphasis has been put in Sibyll 2.3 on model features that are related to the production of muons in the $\mathrm{GeV}$ energy range.

The energy transfer from the hadronic core of an air shower to the electromagnetic component by $\pi^{0}$ production is a very important parameter that influences the number of muons arriving at ground. The more energy is kept in each hadronic interaction in the hadronic core of the shower, i.e. given to hadrons that interact again or decay into secondaries that include muons, the larger is the number of muons at ground. The importance of baryon-antibaryon pair-production in this respect had been noted already in 1973 [118]. Thanks to baryon number conservation, all baryons produced in a shower propagate and re-interact until their energy is too low for hadronic particle production. The multiplicity of secondary baryons is small and, hence, secondary baryons are unimportant in the first generations of an air shower. They serve, however, as a very efficient source of secondary pions at low energy when the charged pions forming the bulk of the hadronic core begin to decay, see Fig. 8. Enhanced baryon pairproduction leads to an enhancement of the number of lowenergy muons in air showers. This has been demonstrated in a detailed study made with EPOS [119]. Simulations

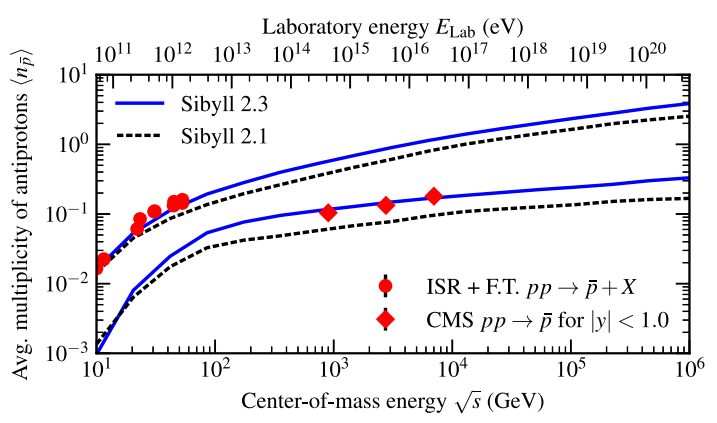

Figure 9. Multiplicity of secondary antiprotons in $p p$ collisions. Shown are results extrapolated to the full phase space of secondary particles (upper curves and points) and data for a central rapidity window (lower curves and points).

show that baryon-induced sub-showers produce about $30 \%$ more muons than pion showers. Indeed, the average number of produced baryon pairs is too low in Sibyll 2.1 and had to be re-tuned, see Fig. 9.

Another aspect of hadronic interactions of direct relevance to muon production is the production of hadronic resonances of high energy in the fragmentation of beam remnants [120]. The surprising experimental observation is that the leading particle in $\pi^{ \pm} p$ interactions is often a $\rho^{0}$ meson instead of a $\pi^{0}$. In contrast to the $\pi^{0}$, the $\rho^{0}$ meson decays directly to two charged pions and the energy of the leading particle is kept in the hadronic shower component [121]. The enhanced production of leading $\rho^{0}$ mesons is not reproduced in models that rely on string fragmentation.

In string fragmentation models, the production ratio of states of different spin and the same valence quarks is given by a model parameter and independent of the production kinematics. This can be seen in Fig. 10 (top). To a good approximation there is a constant ratio between the $\pi^{0}$ and $\rho^{0}$ curves in Sibyll 2.1. The data show a very different behavior. Adding the possibility to have excited states formed by the remnant of a hadron in an interaction allows the implementation of an enhanced production of $\rho^{0}$ mesons in forward direction. This is shown for Sibyll 2.3 in Fig. 10 (bottom).

The impact of the tuned baryon-antibaryon and leading $\rho^{0}$ production on the number of muons at ground is shown in Fig. 11. The Sibyll predictions change by a factor of 1.4 at low energy and 1.6 high energy. Now Sibyll is the model that predicts the highest muon multiplicity in air showers. From these results it is clear that measurements of baryon pair and leading $\rho^{0}$ production will be needed for pion-nucleus interactions to make the prediction of muon numbers in air showers more reliable, see [122].

Finally, for completeness, it should be mentioned that a phenomenological treatment of charm production was implemented in Sibyll 2.3 - based on the approach developed in Ref. [123] - and tuned to fixed-target and LHC data. A realistic model for charm production is very important for calculating prompt lepton production in the atmosphere [8, 124]. The Sibyll predictions for atmospheric lepton fluxes will be discussed in detail in Ref. [125].

\section{The future}

No attempt will be made to predict the future development of Sibyll. Only a number of general observations will 

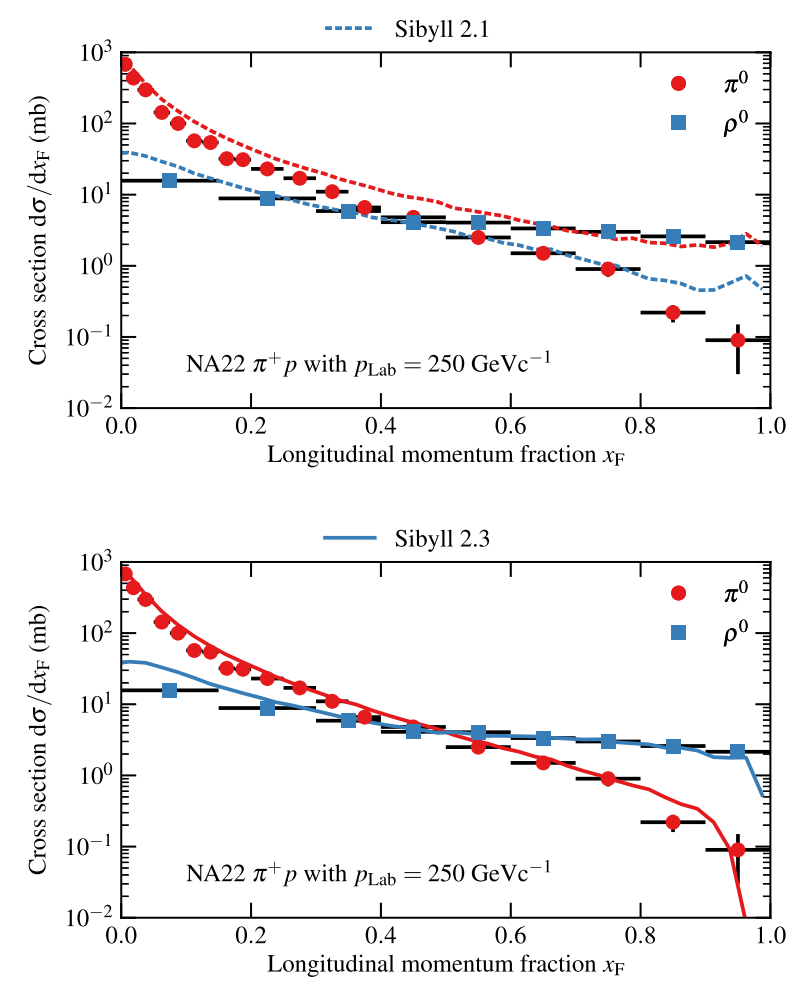

Figure 10. Inclusive $\pi^{0}$ and $\rho^{0}$ production in $\pi^{+} p$ interactions at $250 \mathrm{GeV}$ lab momentum. The top panel shows the predictions of Sibyll 2.1 which was not tuned to describe these data sets. The results of Sibyll 2.3 are shown in the bottom panel after extending the treatment of hadron remnants in the model.

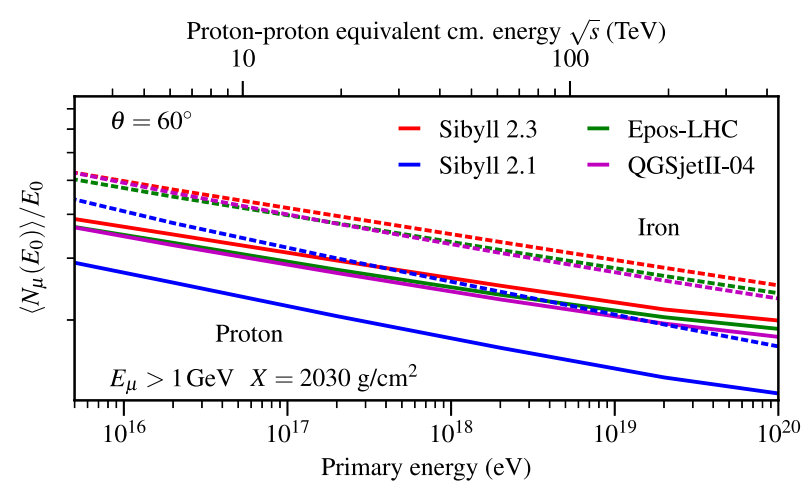

Figure 11. Mean number of muons in air showers of $60^{\circ}$ zenith angle as observed at sea level. The muon energy threshold is $1 \mathrm{GeV}$.

be pointed out focusing on open questions and possible strategies for finding answers.

\subsection{High-precision data and model predictions}

In the last decade an exceptionally large number of minimum bias measurements with very small systematic uncertainties have been published by LHC and fixedtarget experiments. The LHC as an energy frontier has pushed particle production measurements into the energy region in which it is dominated by minijets and collective effects have been found not only in heavy ion but also proton-proton collisions. The awareness of the need for minimum bias measurements with light nuclei has increased and many different fixed-target data sets were published, for example, see [126-129]. Moreover, model builders have also profited from the need of long-baseline neutrino experiments for high-statistics measurements of particle production using different targets including light nuclei [130-133].

This large number of new data has triggered the development of a new generation of hadronic interaction models, often referred to as post-LHC models, which are of much higher quality than the previous generation. At the same time the existence of these rich and very diverse data sets has caused a dilemma for builders of hadronic interaction models. With the lack of not being able to calculate particle production from first principles it cannot be expected that any model, as sophisticated as it might be, will describe all the data reasonably well and provide a consistent view of hadronic interactions.

In light of the large amount of accelerator data available the careful selection of data sets used for optimizing hadronic interaction models for cosmic ray interactions becomes more and more important. Selecting the data according to the covered phase space and their relevance to air shower physics is an important first step. But even the best selection of data used for tuning has its limitations because many data sets provide only indirect information on forward particle production, if at all. There are further limitations stemming from the complexity of the physics of hadronic multiparticle production. For example, multiplicities and transverse momentum spectra of particles in heavy ion collisions should not be used for the tuning of models that do not include a treatment of collective effects such as transverse flow. While such collective effects will probably not be important in air shower physics, they will have to be considered for maximum use of the information provided by LHC data.

The advantage of restricting Sibyll to the modeling of phenomena needed for understanding extensive air showers and, hence, being a very simple and flexible model, turns here to a disadvantage, limiting the number of data sets it can be compared to. With the amount of highly specialized measurements continuously increasing it will be more and more difficult to tune the model to these data sets without introducing new phenomena in the model even if these are not really needed for air shower simulation. One solution to this problem would be, of course, the measurement of forward particle distributions, integrated over transverse momentum and covering large phase space ranges. Those distributions could be used directly for model tuning and air shower simulation.

\subsection{Conceptual problems and open questions}

Theoretical approaches and approximations for describing particle production are available for different energy regions and kinematic regimes. These include resonance and effective field theories close to the particle production threshold, Regge theory [22] for low-energy and low-pt processes, perturbative QCD combined with collinear [134] or $k_{\perp}$ factorization [135] for hard processes in different limits of $\log \left(p_{\perp}^{2} / \Lambda_{\mathrm{QCD}}^{2}\right)$ vs. $\log (1 / x)$, the Color Glass Condensate approach [136] for high parton densities, and hydrodynamics of the Quark-Gluon plasma [137,138] at very high energy densities. Typically, hadronic interaction models include only a small number of these approaches and the transition is between these kinematic regimes and covered phase space regions is implemented in a pragmatic way, driven by the goal of reaching a good 


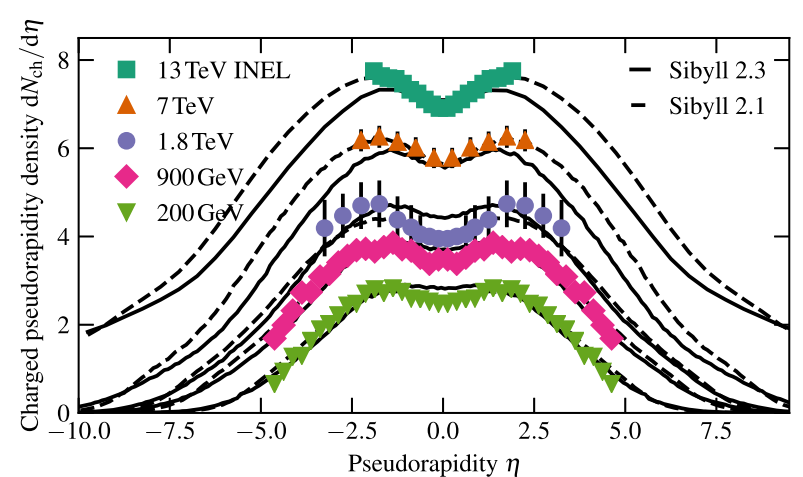

Figure 12. Pseudorapidity distribution of charged particles. Collider data from CMS, CDF and UA5 [43,87,139,140]. are compared to simulations made with Sibyll versions 2.1 and 2.3. At high energy Sibyll 2.3 systematically predicts a lower particle density in forward and backward directions than measured.

description of data. More theoretical work is needed to better understand the transition between the theoretical treatments.

One prominent example of the need to combine two different approaches is the transition from soft to hard processes. At low energy, in the energy range of fixedtarget experiments, multiparticle production is dominated by soft processes and hadronic degrees of freedom are most appropriate for model building. In the energy range from $\sqrt{s} \sim 100-1000 \mathrm{GeV}$, a transition to partonic degrees of freedom takes place. And at high energies, most clearly seen at LHC for the first time, parton-parton interactions are dominating large parts of phase space in multiparticle production. Tuning a model and obtaining a very good description of low-energy data does not automatically imply a good description of high-energy data.

The assumptions on the description of the transition from hard to semi-hard and then to soft processes play an important role at high energy and are an essential element of model building. This is illustrated in Fig. 12 showing the pseudorapidity distribution of charged particles over a wide range of collision energies. At low energy soft interactions are still producing the bulk of the particles. This is no longer the case at LHC energies. The discrepancies between data and Sibyll 2.3 at $|\eta| \sim 2$ indicate some shortcoming in the modeling of the transition between soft and hard processes, in particular of the parton densities at very low $x$, or possibly of the underlying soft processes in the presence of hard parton-parton interactions. That these shortcomings do not necessarily affect the very forward direction is demonstrated in Fig. 13, in which the forward neutron production at LHC is compared to Sibyll predictions. There the improvement of data description with Sibyll 2.3 wrt. version 2.1 is clearly visible.

In Sibyll, the geometric criterion (6) is used for defining a threshold for the applicability of the simple multiple-scattering model. A straightforward generalization to nuclei is the use of the sum of the local parton densities of all nucleons in Eq. (6), taken at the relevant impact parameter of the interaction. This means that, in general, the transverse momentum threshold for hard interactions should vary from collision to collision, and the parameters for soft interactions should change too. For comparison, in EPOS, the parton densities are modified
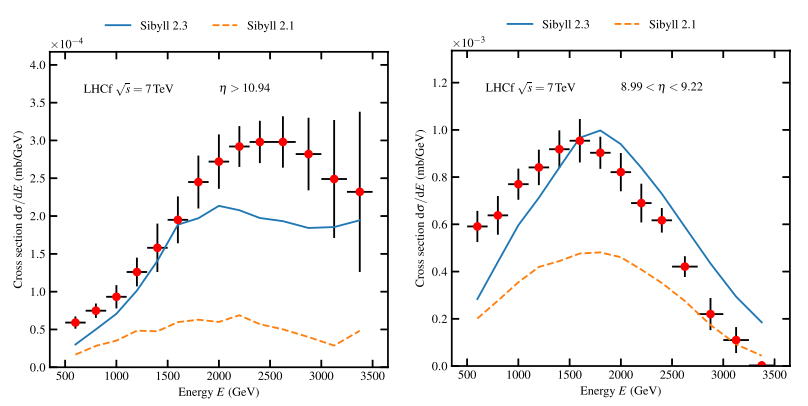

Figure 13. Leading neutron production as measured with the LHCf experiment [142]. The data are compared with the predictions of two versions of Sibyll.

at low $x$ by hand, i.e. the number of partons is reduced, in dependence on the local energy density, irrespective of how many nucleons are participating in a collision [100]. In QGSJET II (soft) pomeron-pomeron interactions (socalled enhanced pomeron graphs) are re-summed to all order, leading to a rather involved theoretical description of hadronic interactions $[104,105,141]$. All these approaches for implementing a transition from hard to soft processes imply the violation of QCD factorization at semi-hard, minijet scales while preserving factorization for high- $p_{\perp}$ processes. The diversity of the approaches is a sign of our lack of understanding semi-hard processes.

It has been speculated already for a long time that central particle production will ultimately also change leading particle distributions once the scattering process the black disk limit [143]. This effect is analogous to the suppression of leading particle production in dependence on the number of participating nucleons (i.e. centrality) in hadron-nucleus interactions. It will be important to demonstrate this effect at LHC by, for example, combining the central data taken by ATLAS with that of the forward experiment LHC on an event-by-event basis. Furthermore, because we have little knowledge on the physics relation between central and forward particle production, we still cannot reliably predict the leading particle distributions in hadronic interactions involving nuclei even if these distributions were known (measured) in $p p$ and $\pi p$ interactions. Only the direct measurement of proton interactions with light nuclei can help to reduce these uncertainties. With oxygen being used as carrier ion for heavy ion injection, $p O$ interactions should be a technically feasible option at LHC without too much accelerator tuning needed.

The authors thank their colleagues of the Pierre Auger, IceCube and KASCADE-Grande Collaborations for many inspiring discussions. In particular, the very fruitful interaction with Tanguy Pierog is gratefully acknowledged. We also thank Bryan Pattison for comments on the manuscript. This work is supported in part by the German Ministry of Education and Research (BMBF), grant No. 05A14VK1, and the Helmholtz Alliance for Astroparticle Physics (HAP), which is funded by the Initiative and Networking Fund of the Helmholtz Association.

\section{References}

[1] B. Rossi, K. Greisen, Rev. Mod. Phys. 13, 240 (1941)

[2] J. Nishimura, Handbuch der Physik 46/2, 1 (1965)

[3] P. Lipari, Astropart. Phys. 1, 195 (1993) 
[4] T.K. Gaisser, R. Engel, E. Resconi, Cosmic Rays and Particle Physics, 2nd edn. (Cambridge University Press, 2016), ISBN 9780521016469

[5] L.G. Dedenko, Proc. of 9th Int. Cosmic Ray Conf., London 1, 662 (1965)

[6] T.K. Gaisser, R.J. Protheroe, K.E. Turver, T.J.L. McComb, Rev. Mod. Phys. 50, 859 (1978)

[7] G. Bossard et al., Phys. Rev. D63, 054030 (2001), hep-ph/0009119

[8] A. Fedynitch, R. Engel, T.K. Gaisser, F. Riehn, T. Stanev, EPJ Web Conf. 99, 08001 (2015), Proc. of 18th Int. Symposium on Very High Energy Cosmic Ray Interactions (ISVHECRI 2014) Geneva, Switzerland, August 18-22, 2014, 1503.00544

[9] H.J. Drescher, G.R. Farrar, Phys. Rev. D67, 116001 (2003), astro-ph/0212018

[10] T. Bergmann et al., Astropart. Phys. 26, 420 (2007), astro-ph/0606564

[11] S.J. Sciutto (1999), astro-ph/9911331

[12] S.J. Sciutto (2001), astro-ph/0106044

[13] D. Heck, J. Knapp, J.N. Capdevielle, G. Schatz, T. Thouw, Wissenschaftliche Berichte, Forschungszentrum Karlsruhe FZKA 6019 (1998)

[14] K. Kasahara et al., http: //cosmos.n.kanagawau.ac.jp/cosmosHome

[15] A.M. Hillas, Proc. of 17th Int. Cosmic Ray Conf., Paris 8, 193 (1981)

[16] A.M. Hillas, Nucl. Phys. Proc. Suppl. 52B, 29 (1997)

[17] J. Engel, T.K. Gaisser, T. Stanev, P. Lipari, Phys. Rev. D46, 5013 (1992)

[18] R.S. Fletcher, T.K. Gaisser, P. Lipari, T. Stanev, Phys. Rev. D50, 5710 (1994)

[19] E.J. Ahn, R. Engel, T.K. Gaisser, P. Lipari, T. Stanev, Phys. Rev. D 80, 094003 (2009), 0906.4113

[20] F. Riehn, R. Engel, A. Fedynitch, T.K. Gaisser, T. Stanev, PoS (ICRC2015), 558 (2015), 1510.00568

[21] F. Riehn, R. Engel, A. Fedynitch, T.K. Gaisser, T. Stanev, EPJ Web Conf. 99, 12001 (2015), 1502.06353

[22] P.D.B. Collins, An Introduction to Regge Theory and High-Energy Physics (Cambridge Univ. Pr., 1977)

[23] G.F. Chew, S.C. Frautschi, Phys. Rev. Lett. 8, 41 (1962)

[24] E. Klempt, A. Zaitsev, Phys. Rept. 454, 1 (2007), 0708.4016

[25] R.P. Feynman, Phys. Rev. Lett. 23, 1415 (1969)

[26] Z. Koba, H.B. Nielsen, P. Olesen, Nucl. Phys. B40, 317 (1972)

[27] J. Benecke, T.T. Chou, C.N. Yang, E. Yen, Phys. Rev. 188, 2159 (1969)

[28] A.H. Mueller, Phys. Rev. D2, 2963 (1970)

[29] M. Gell-Mann, Phys. Lett. 8, 214 (1964)

[30] G. Zweig, preprint CERN-TH-401 (1964)

[31] R.P. Feynman, Proc. of High Energy Collisions: Third International Conference at Stony Brook pp. 237-249 (1969), N.Y. Gordon \& Breach

[32] J.D. Bjorken, E.A. Paschos, Phys. Rev. 185, 1975 (1969)
[33] S.D. Drell, T.M. Yan, Annals Phys. 66, 578 (1971)

[34] H. Fritzsch, M. Gell-Mann, H. Leutwyler, Phys. Lett. B47, 365 (1973)

[35] B.L. Combridge, J. Kripfganz, J. Ranft, Phys. Lett. B70, 234 (1977)

[36] B.L. Combridge, Nucl. Phys. B151, 429 (1979)

[37] J.F. Owens, E. Reya, M. Gluck, Phys. Rev. D18, 1501 (1978)

[38] W. Ochs, Nucl. Phys. B118, 397 (1977)

[39] G. Altarelli, G. Parisi, Nucl. Phys. B126, 298 (1977)

[40] P.D.B. Collins, A.D. Martin, Rept. Prog. Phys. 45, 335 (1982)

[41] G.J. Alner et al. (UA5), Z. Phys. C32, 153 (1986)

[42] C. Augier et al. (UA4/2), Phys. Lett. B344, 451 (1995)

[43] G.J. Alner et al. (UA5), Z. Phys. C33, 1 (1986)

[44] G.J. Alner et al. (UA5), Phys. Lett. B138, 304 (1984)

[45] G.J. Alner et al. (UA5), Phys. Rept. 154, 247 (1987)

[46] C. Albajar et al. (UA1), Nucl. Phys. B309, 405 (1988)

[47] L.V. Gribov, E.M. Levin, M.G. Ryskin, Phys. Rept. 100, 1 (1983)

[48] T.K. Gaisser, F. Halzen, Phys. Rev. Lett. 54, 1754 (1985)

[49] G. Pancheri, Y.N. Srivastava, Phys. Lett. B182, 199 (1986)

[50] L. Durand, H. Pi, Phys. Rev. Lett. 58, 303 (1987)

[51] L. Ametller, D. Treleani, Int. J. Mod. Phys. A3, 521 (1988)

[52] A. Capella, J. Tran Thanh Van, J. Kwiecinski, Phys. Rev. Lett. 58, 2015 (1987)

[53] J.H. Weis, Acta Phys. Polon. B7, 851 (1976)

[54] K. Werner, Phys. Rep. 232, 87 (1993)

[55] T.K. Gaisser, T. Stanev, Phys. Lett. B219, 375 (1989)

[56] T.K. Gaisser, U. Sukhatme, G.B. Yodh, Phys. Rev. D36, 1350 (1987)

[57] L. Durand, H. Pi, Phys. Rev. D40, 1436 (1989)

[58] L. Durand, H. Pi, Phys. Rev. D43, 2125 (1991)

[59] J. Dias de Deus, J. Kwiecinski, Phys. Lett. B196, 537 (1987)

[60] B. Margolis, P. Valin, M.M. Block, F. Halzen, R.S. Fletcher, Phys. Lett. B213, 221 (1988)

[61] T.K. Gaisser, F. Halzen, A.D. Martin, Phys. Lett. B166, 219 (1986)

[62] J. Dias de Deus, J. Kwiecinski, M. Pimenta, Phys. Lett. B202, 397 (1988)

[63] K. Alpgard et al. (UA5), Phys. Lett. B123, 361 (1983)

[64] K.A. Ter-Martirosyan, Phys. Lett. B44, 377 (1973)

[65] A. Capella, U. Sukhatme, C.I. Tan, J. Tran Thanh Van, Phys. Rept. 236, 225 (1994)

[66] A.B. Kaidalov, Phys. Atom. Nucl. 66, 1994 (2003)

[67] T. Sjöstrand, M. van Zijl, Phys. Rev. D36, 2019 (1987)

[68] B. Andersson, G. Gustafson, G. Ingelman, T. Sjostrand, Phys. Rept. 97, 31 (1983)

[69] T. Sjöstrand, Int. J. Mod. Phys. A3, 751 (1988)

[70] T.K. Gaisser et al., Phys. Rev. D47, 1919 (1993) 
[71] R.J. Glauber, G. Matthiae, Nucl. Phys. B21, 135 (1970)

[72] A. Etchegoyen et al. (1996), FERMILAB-PUB-96024, http://www.auger.org/admin/Design Report/

[73] A. Aab et al. (Pierre Auger), Nucl. Instrum. Meth. A798, 172 (2015), 1502.01323

[74] E. Eichten, I. Hinchliffe, K.D. Lane, C. Quigg, Rev. Mod. Phys. 56, 579 (1984), [Addendum: Rev. Mod. Phys.58,1065(1986)]

[75] D.W. Duke, J.F. Owens, Phys. Rev. D30, 49 (1984)

[76] T. Antoni et al. (KASCADE), Nucl. Instrum. Meth. A513, 490 (2003)

[77] M.L. Good, W.D. Walker, Phys. Rev. 120, 1857 (1960)

[78] V.A. Abramovski, V.N. Gribov, O.V. Kancheli, Sov. J. Nucl. Phys. 18, 308 (1974)

[79] M. Glück, E. Reya, A. Vogt, Z. Phys. C53, 651 (1992)

[80] M. Glück, E. Reya, A. Vogt, Z. Phys. C67, 433 (1995)

[81] E.M. Levin, M.G. Ryskin, Phys. Rep. 189, 267 (1990)

[82] V.N. Gribov, Sov. Phys. JETP 26, 414 (1968), [Zh. Eksp. Teor. Fiz.53,654(1967)]

[83] R. Engel, Z. Phys. C66, 203 (1995)

[84] R. Engel, J. Ranft, Phys. Rev. D54, 4244 (1996), hep-ph/9509373

[85] J. Ranft, Phys. Rev. D51, 64 (1995)

[86] S. Roesler, R. Engel, J. Ranft, Proc of 27th Int. Cosmic Ray Conf., Hamburg 2, 439 (2001)

[87] F. Abe et al. (CDF), Phys. Rev. D41, 2330 (1990)

[88] R. Harr et al., Phys. Lett. B401, 176 (1997), hep-ex/9703002

[89] K. Alpgard et al. (UA5), Phys. Lett. B112, 183 (1982)

[90] R. Engel, T.K. Gaisser, T. Stanev, P. Lipari, Proc. of 26th Int. Cosmic Ray Conf., Salt Lake City 1, 415 (1999)

[91] D. d'Enterria, R. Engel, T. Pierog, S. Ostapchenko, K. Werner, Astropart. Phys. 35, 98 (2011), 1101.5596

[92] G. Aad et al. (ATLAS), Nucl. Phys. B889, 486 (2014), 1408.5778

[93] M. Aaboud et al. (ATLAS), Phys. Rev. Lett. 117, 182002 (2016), 1606.02625

[94] B. Abelev et al. (ALICE), Eur. Phys. J. C73, 2456 (2013), 1208.4968

[95] S. Chatrchyan et al. (CMS), Phys. Lett. B722, 5 (2013), 1210.6718

[96] G. Antchev et al. (TOTEM), Europhys. Lett. 95, 41001 (2011), 1110.1385

[97] G. Antchev et al. (TOTEM), Phys. Rev. Lett. 111, 012001 (2013)

[98] P. Abreu et al. (Pierre Auger), Phys. Rev. Lett. 109, 062002 (2012), 1208.1520

[99] F.M. Liu, J. Aichelin, K. Werner, M. Bleicher, Phys. Rev. C69, 054002 (2004), hep-ph/0307008

[100] K. Werner, F.M. Liu, T. Pierog, Phys. Rev. C74, 044902 (2006), hep-ph/0506232

[101] K. Werner, T. Pierog, AIP Conf. Proc. 928, 111 (2007), 0707.3330

[102] N.N. Kalmykov, S.S. Ostapchenko, Sov. J. Nucl. Phys. 50, 315 (1989)
[103] N.N. Kalmykov, S.S. Ostapchenko, A.I. Pavlov, Nucl. Phys. Proc. Suppl. 52B, 17 (1997)

[104] S. Ostapchenko, Phys. Rev. D74, 014026 (2006), hep-ph/0505259

[105] S. Ostapchenko, Phys. Lett. B636, 40 (2006), hep-ph/0602139

[106] F. Riehn et al. (2017), in preparation

[107] N.A. Amos et al. (E710), Phys. Rev. Lett. 63, 2784 (1989)

[108] F. Abe et al. (CDF), Phys. Rev. D50, 5550 (1994)

[109] C. Avila et al. (E811), Phys. Lett. B445, 419 (1999)

[110] T. Abu-Zayyad et al. (HiRes-MIA), Astrophys. J. 557, 686 (2001), astro-ph/0010652

[111] R.U. Abbasi et al. (HiRes), Astrophys. J. 622, 910 (2005), astro-ph/0407622

[112] A. Aab et al. (Pierre Auger), Phys. Rev. D90, 122005 (2014), 1409.4809

[113] R. Engel, D. Heck, T. Pierog, Ann. Rev. Nucl. Part. Sci. 61, 467 (2011)

[114] T. Antoni et al. (KASCADE), Astropart. Phys. 24, 1 (2005), astro-ph/0505413

[115] R. Engel (Pierre Auger), Proc. of 30th Int. Cosmic Ray Conf., Merida 4, 385 (2007), arXiv:0706.1921 [astro-ph]

[116] A. Aab et al. (Pierre Auger), Phys. Rev. D91, 032003 (2015), 1408.1421

[117] A. Aab et al. (Pierre Auger), Phys. Rev. Lett. 117, 192001 (2016), 1610.08509

[118] P.K.F. Grieder, Proc. of 13th Int. Cosmic Ray Conf., Denver 4, 2467 (1973)

[119] T. Pierog, K. Werner, Phys. Rev. Lett. 101, 171101 (2008), astro-ph/0611311

[120] H.J. Drescher, Phys. Rev. D77, 056003 (2007), arXiv:0712.1517 [hep-ph]

[121] S. Ostapchenko, EPJ Web Conf. 52, 02001 (2013)

[122] A. Hervé et al. (NA61) (2017), these proceedings

[123] E.J. Ahn, R. Engel, T.K. Gaisser, P. Lipari, T. Stanev (2011), Proc. of 16th Int. Symposium on Very High Energy Cosmic Ray Interactions (ISVHECRI 2010): Batavia, Illinois, USA, June 28-July 2, 2010, 1102.5705

[124] T.K. Gaisser, M. Honda, Ann. Rev. Nucl. Part. Sci. 52, 153 (2002), hep-ph/0203272

[125] A. Fedynitch et al. (2017), in preparation

[126] C. Alt et al. (NA49), Eur. Phys. J. C45, 343 (2006), hep-ex/0510009

[127] C. Alt et al. (NA49), Eur. Phys. J. C49, 897 (2007), hep-ex/0606028

[128] T. Anticic et al. (NA49), Eur. Phys. J. C68, 1 (2010), 1004.1889

[129] B. Baatar et al. (NA49), Eur. Phys. J. C73, 2364 (2013), 1207.6520

[130] N. Abgrall et al. (NA61/SHINE), Phys. Rev. C 84, 034604 (2011), 1102.0983

[131] N. Abgrall et al. (NA61/SHINE), Phys. Rev. C85, 035210 (2012), 1112.0150

[132] N. Abgrall et al. (NA61/SHINE), Phys. Rev. C89, 025205 (2014), 1309.1997

[133] N. Abgrall et al. (NA61/SHINE), Eur. Phys. J. C74, 2794 (2014), 1310. 2417

[134] J.C. Collins, D.E. Soper, G.F. Sterman, Nucl. Phys. B261, 104 (1985)

[135] S. Catani, M. Ciafaloni, F. Hautmann, Nucl. Phys. B366, 135 (1991) 
[136] F. Gelis, E. Iancu, J. Jalilian-Marian, R. Venugopalan, Ann. Rev. Nucl. Part. Sci. 60, 463 (2010), 1002. 0333

[137] D.H. Rischke, Prog. Part. Nucl. Phys. 52, 197 (2004), nucl-th/0305030

[138] E. Shuryak, Prog. Part. Nucl. Phys. 62, 48 (2009), 0807.3033

[139] V. Khachatryan et al. (CMS), Phys. Lett. B751, 143 (2015), 1507.05915
[140] V. Khachatryan et al. (CMS), Phys. Rev. Lett. 105, 022002 (2010), 1005. 3299

[141] S. Ostapchenko, Phys. Rev. D81, 114028 (2010), 1003.0196

[142] O. Adriani et al. (LHCf), Phys. Lett. B750, 360 (2015), 1503.03505

[143] H.J. Drescher, A. Dumitru, M. Strikman, Phys. Rev. Lett. 94, 231801 (2005), hep-ph/0408073 